\title{
First step towards a non-adiabatic description of the fission process based on the Generator Coordinate Method
}

\author{
R. Bernard ${ }^{1}$, H. Goutte ${ }^{1}$, D. Gogny ${ }^{2}$, N. Dubray ${ }^{1}$, and D. Lacroix ${ }^{3}$ \\ ${ }^{1}$ CEA, DAM, DIF, F - 91297 Arpajon, France \\ 2 Lawrence Livermore National Laboratory, Livermore, California 94550, USA \\ ${ }^{3}$ GANIL, CEA/DSM - CNRS/IN2P3, F-14076 Caen, France
}

\begin{abstract}
Among the different theoretical approaches able to describe fission, microscopic ones can help us in the understanding of this process, as they have the advantage of describing the nuclear structure and the dynamics in a consistent manner. The sole input of the calculations is the nucleon-nucleon interaction. Such a microscopic time-dependent and quantum mechanical formalism has already been used, based on the Gaussian Overlap Approximation of the Generator Coordinate Method with the adiabatic approximation, to analyze the collective dynamics of low-energy fission in ${ }^{238} \mathrm{U}$ [1]. However, at higher energies, a few $\mathrm{MeV}$ above the barrier, the adiabatic approximation doesn't seem valid anymore. Indeed, manifestations of proton pair breaking have been observed in ${ }^{238} \mathrm{U}$ and ${ }^{239} \mathrm{U}$ for an excitation energy of $2.3 \mathrm{MeV}$ above the barrier [2-4]. Taking the intrinsic excitations into account during the fission process will enable us to determine the coupling between collective and intrinsic degrees of freedom, in particular from saddle to scission. Guidelines of the new formalism under development are presented and some preliminary results on overlaps between non excited and excited states are discussed.
\end{abstract}

\section{Introduction}

The Generator Coordinate Method (GCM) is a very usefull approach to study large amplitude collective modes, in particular the fission process. Adiabatic GCM calculations based on the Gaussian Overlap Approximation (GOA) have shown a rather good agreement between calculated and measured fragment mass, and fragment kinetic energy distributions for low-energy fission [1]. However there are some evidences that intrinsic excitations may play a role during the fission process. Indeed, some experiments show that proton pair breakings occur for an excitation energy of $2.3 \mathrm{MeV}$ above the barrier in ${ }^{238} \mathrm{U}$ and ${ }^{239} \mathrm{U}$. First, the total kinetic energy suddenly drops for an excitation energy slightly higher than $2.3 \mathrm{MeV}[2,3]$ and second, the proton odd even effect decreases exponentially to zero for the same excitation energy [4].

Some theoretical calculations have already studied nonadiabatic effects during the fission process, most of them being based on a semi-classical formalism such as FokkerPlank equations [5-7], or Hamilton equations with one body dissipation and two-body viscosity [8,9], or Langevin equations $[10,11]$. In addition, a microscopic approach based on transport theories has recently been proposed by K. Dietrich et al [12].

Here the extended formalism under development is sketched, where excited states are taken into account in the framework of the Generator Coordinate Method. A few pioneering calculations along this line have already been performed in the past [13-15]. Selection rules for the choice of the pertinent intrinsic excitations are presented. Prelimi- nary results on overlaps between deformed excited and non excited basis states are discussed.

\section{Non-adiabatic Generator Coordinate Method}

In the non-adiabatic version of the time-dependent Generator Coordinate Method, the nuclear state is defined as

$$
|\Psi(t)\rangle=\sum_{i} \int d q f_{i}(q, t)\left|\Phi_{i}(q)\right\rangle,
$$

where the time dependent weight functions $f_{i}(q, t)$ are obtained through

$$
\frac{\partial}{\partial f_{i}^{*}\left(q^{\prime}, t^{\prime}\right)} \int_{t_{1}}^{t_{2}}\left\langle\Psi(t)\left|\hat{H}-i \hbar \frac{\partial}{\partial t}\right| \Psi(t)\right\rangle d t=0
$$

In Eq. (1), $\hat{H}$ is the nuclear Hamiltonian, and $\left|\Phi_{i}(\{q\})\right\rangle$ is a set of static states depending on the collective variable $q$ and on single particle excitations $i$, where $\mathrm{i}=0$ stands for the lowest-energy state.

The variational principle (Eq. (2)) leads to the generalized Hill-Wheeler equation :

$$
\sum_{i} \int d q\left(\left\langle\Phi_{j}\left(q^{\prime}\right)|\hat{H}| \Phi_{i}(q)\right\rangle-i \hbar \frac{\partial}{\partial t}\left\langle\Phi_{j}\left(q^{\prime}\right) \mid \Phi_{i}(q)\right\rangle\right) f_{i}(q, t)=0 .
$$

This is an Open Access article distributed under the terms of the Creative Commons Attribution-Noncommercial License, which permits unrestricted use, distribution, and reproduction in any noncommercial medium, provided the original work is properly cited. 
In the usual adiabatic case, without intrinsic excitations, the adiabatic nuclear state $\left|\Psi_{\text {adiab }}(t)\right\rangle$ is defined as

$$
\left|\Psi_{\text {adiab }}(t)\right\rangle=\int d q f_{\text {adiab }}(q, t)|\Phi(q)\rangle,
$$

where $|\Phi(q)\rangle$ is the lowest energy state at a fixed $q$. In Refs. [1] and [16] the Gaussian Overlap Approximation has been used to obtain a Schrödinger-like equation :

$$
H_{\text {coll }} g_{a d i a b}(q, t)=i \hbar \frac{\partial}{\partial t} g_{a d i a b}(q, t),
$$

with $g_{\text {adiab }}(q, t)$ the Gauss transform of $f_{\text {adiab }}(q, t)$ and

$$
H_{\text {coll }}=\langle\Phi(q)|\hat{H}| \Phi(q)\rangle-Z P E-\frac{\hbar^{2}}{2} \frac{\partial}{\partial q} \frac{1}{M(q)} \frac{\partial}{\partial q},
$$

where $M(q)$ is the collective mass and $Z P E$ is the zero point energy correction. The Gaussian Overlap Approximation is based on the fact, among others, that the overlap between deformed basis states is indeed, of gaussian shape:

$$
\left\langle\Phi\left(q^{\prime}\right) \mid \Phi(q)\right\rangle=e^{-G\left(q-q^{\prime}\right)^{2}} .
$$

The validity of the GOA has been discussed in $[17,18]$. Here the problem deals with the generalization of the GOA to the case where intrinsic excitations are taken into account in the formalism. To solve this problem, the excitations along the fission paths have been first studied and the overlaps between excited states have then been calculated.

\section{Study of the 2 quasi-particle excitations along the fission paths of ${ }^{236} \mathbf{U}$}

Here, the intrinsic deformed states $\left|\Phi_{i=0}(q)\right\rangle \equiv|\Phi(q)\rangle$ are Hartree-Fock-Bogoliubov states obtained through the variational principle

$$
\delta\left\langle\Phi(q)\left|\hat{H}-\lambda_{N} \hat{N}-\lambda_{Z} \hat{Z}-\lambda_{q} \hat{Q}\right| \Phi(q)\right\rangle=0,
$$

where the Lagrange parameters $\lambda_{N}, \lambda_{Z}$, and $\lambda_{q}$ are deduced from

$$
\left\{\begin{array}{l}
\langle\Phi(q)|\hat{N}(\hat{Z})| \Phi(q)\rangle=N(Z) \\
\langle\Phi(q)|\hat{Q}| \Phi(q)\rangle=q .
\end{array}\right.
$$

In the present calculations, $\hat{Q}$ is the axial quadrupole operator defined as

$$
\hat{Q}=\sqrt{\frac{16 \pi}{5}} \sum_{i=1}^{A} r_{i}^{2} Y_{20}=\sum_{i=1}^{A}\left(2 z_{i}^{2}-x_{i}^{2}-y_{i}^{2}\right)
$$

and $\hat{H}$ is the nuclear many-body effective Hamiltonian built with the finite-range effective D1S force of Gogny [19,20]. The HFB equations are solved by expanding the single particle states onto one-center and two-centers axial harmonic oscillator basis. Because calculations are performed in an even-even nucleus for which $\mathrm{K}=0$ - with $\mathrm{K}$ the projection of the spin onto the symmetry axis -, the HFB nuclear states are even under time-reversal symmetry $T$. Furthermore, we restrict the Bogoliubov space by imposing the

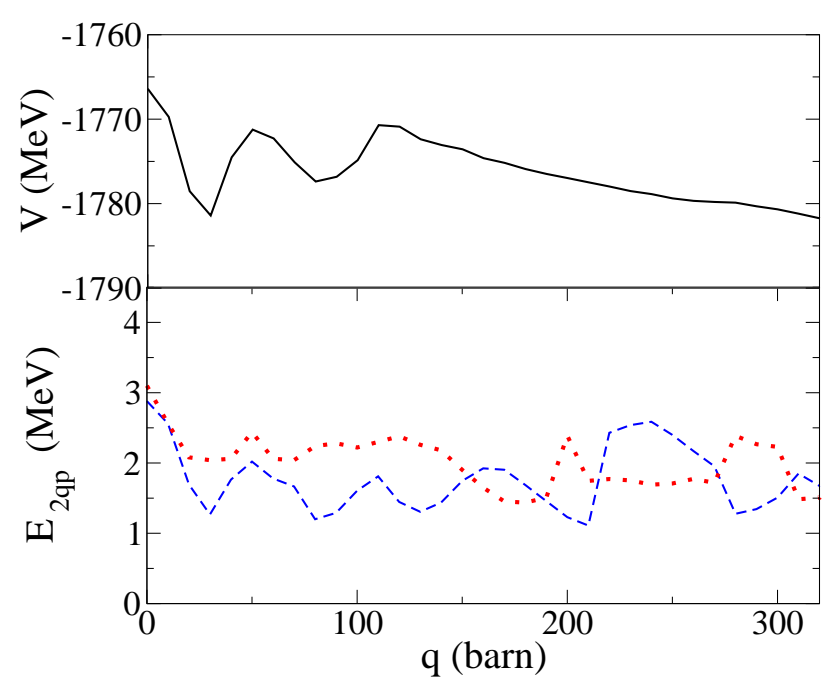

Fig. 1. (Color online) Upper panel: Potential energy along the asymmetric path in ${ }^{236} U$ as a function of the elongation. Lower panel: energy of the lowest neutron (dashed line) and proton (dotted line) two quasi-particle excitation along the asymmetric path.

self-consistent symmetry $\hat{T} \hat{\Pi}_{2}$, where $\hat{\Pi}_{2}$ is the reflection with respect to the $\mathrm{xOz}$ plane.

For the excited states, $\mathrm{K}=0$ two-quasi-particle (2qp) excitations have been selected. The restrictions to $K=0$ quasiparticle states may be justified by the fact that axial symmetry is enforced in the present calculation and that only $\mathrm{K}=0$ excitations may be coupled to the lowest energy $\mathrm{K}=0$ HFB states in even-even nuclei. We define the excited states $\left|\Phi_{i>0}(q)\right\rangle$ through

$$
\left|\Phi_{i}(q)\right\rangle=\eta_{i}^{q+} \eta_{\bar{i}}^{q+}|\Phi(q)\rangle
$$

where $\eta_{i}^{q+}$ is the creation operator of a quasi-particle in the state $\mathrm{i}$, and $\bar{i}$ is the time-reversed state of $\mathrm{i}$.

Since the purpose of this work is the study of the fission process at rather low energy, the other kinds of $\mathrm{N}$ quasiparticle excitations (4qp, 6qp...) have been neglected. Moreover, a cut off at $5 \mathrm{MeV}$ has been used on the 2 quasiparticle excitation energy. Here, no self-consistent blocking is performed, and the HFB vacuum is not polarized by the excited quasi-particles. Thus, by construction, for a given value of the collective variable q, each $2 q p$ state is orthogonal to the 0qp HFB state and to all the other 2qp states, that is $\left\langle\Phi_{i}(q) \mid \Phi_{j}(q)\right\rangle=0$, for $i \neq j$.

Then, by considering such excited intrinsic states, the static basis used to define the non adiabatic GCM state (see Eq. (1)) is enlarged with orthogonal states. The main drawback is that the mean particle number of the 2qp states can deviate from $N_{0}$ and $Z_{0}$, with $N_{0}=\langle\Phi(q)|\hat{N}| \Phi(q)\rangle$ and $Z_{0}=\langle\Phi(q)|\hat{Z}| \Phi(q)\rangle$. Here, the quasi-particle excitations are selected such as :

$$
\left\langle\phi_{i}|\hat{N}(\hat{Z})| \phi_{j}\right\rangle=\langle\phi|\hat{N}(\hat{Z})| \phi\rangle+\Delta N(Z),
$$

with

$$
\Delta N(Z) \leq 1
$$


Calculations have been performed in ${ }^{236} \mathrm{U}$. The potential energy and the excitation energies of the lowest 2qp neutron and $2 \mathrm{qp}$ proton states are plotted as functions of the quadrupole deformation along the asymmetric path on Fig. 1. We clearly see that these energies are varying in the range 1 to $3 \mathrm{MeV}$ and is equal to $2 \mathrm{MeV}$ in average for both neutron and proton excitations. These excitations may be low enough to play a role during the fission process. Indeed, these results have to be compared with those obtained in the experiment of ${ }^{238} \mathrm{U}$ and ${ }^{239} \mathrm{U}$ where proton pair breakings are observed for an excitation energy of 2.3 $\mathrm{MeV}$ above the barrier [2-4].

\section{Calculation of the overlaps}

In Fig. 2 the overlaps $\left\langle\Phi(q) \mid \Phi\left(q^{\prime}\right)\right\rangle$ between HFB states at different deformations are plotted as functions of q-q' for different $\left(q+q^{\prime}\right) / 2$ values corresponding to HFB groundstate, first saddle point, superdeformed minimum, second saddle point and in the descent to scission.

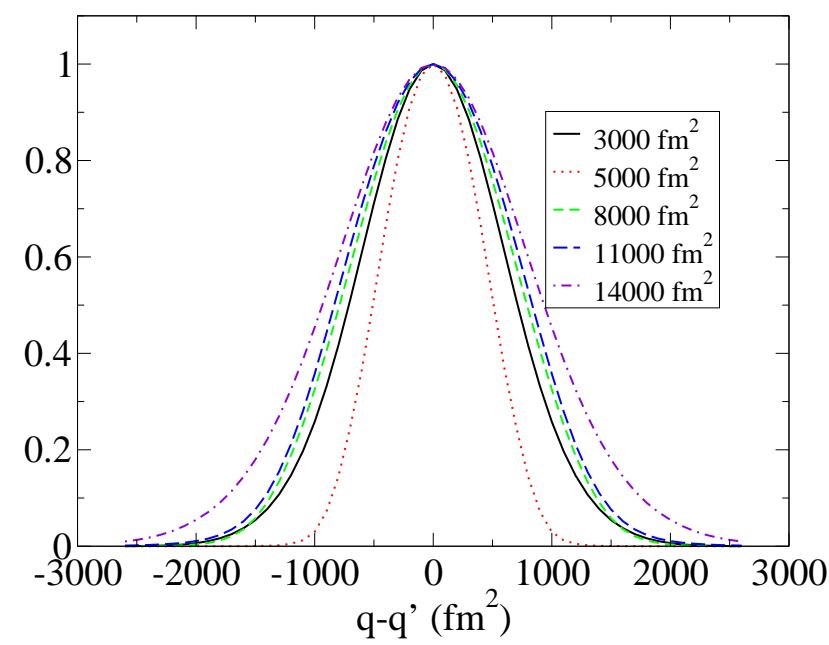

Fig. 2. (Color online) Comparison between the overlaps $\left\langle\Phi(q) \mid \Phi\left(q^{\prime}\right)\right\rangle$ calculated for different values of $\left(q+q^{\prime}\right) / 2$.

At first sight, the overlaps seem to have gaussian shapes as expected. The variation of the width remains small, except for $\left(q+q^{\prime}\right) / 2=5000 \mathrm{fm}^{2}$ where the width is divided by 2 compared to others. The shape of these overlaps will be studied in more details in a near future.

Overlaps $\left\langle\Phi(q)\left|\eta_{\bar{i}}^{q} \eta_{i}^{q} \eta_{i}^{+q^{\prime}} \eta_{\bar{i}}^{+q^{\prime}}\right| \Phi\left(q^{\prime}\right)\right\rangle$ between 2 qp excited states are plotted in Fig. 3 for different qp excitations, namely $K^{\Pi}=1 / 2^{-}, 3 / 2^{-}$, and $5 / 2^{-}$, with $\mathrm{K}$ the projection of the angular momentum on the symmetry axis and $\Pi$ the parity. They are compared with overlaps $\left\langle\Phi(q) \mid \Phi\left(q^{\prime}\right)\right\rangle$ between HFB lowest energy states at $\left(q+q^{\prime}\right) / 2=3000 \mathrm{fm}^{2}$. The more striking feature is that all the widths of the different overlaps are almost the same since all the curves are superimposed. This property will probably be used during

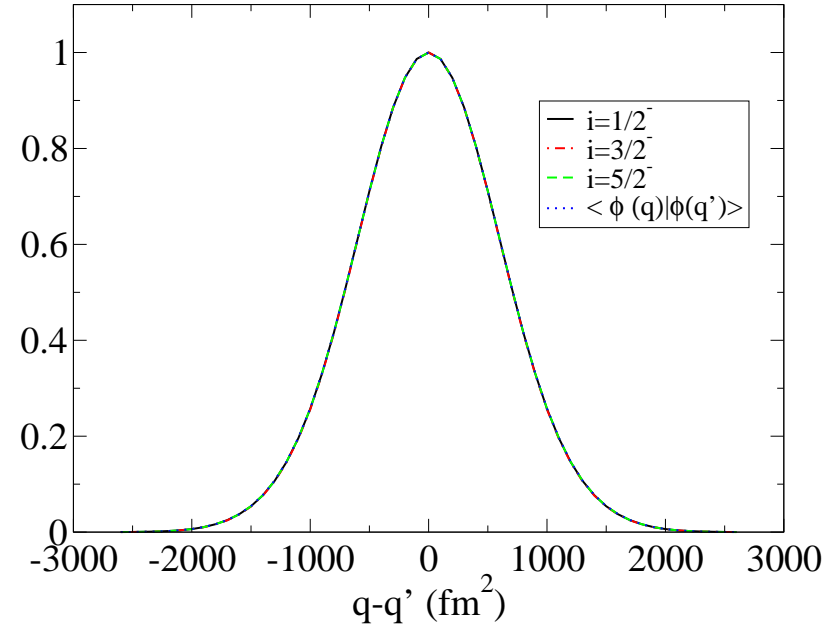

Fig. 3. (Color online) Overlaps $\left\langle\Phi_{i}(q) \mid \Phi_{i}\left(q^{\prime}\right)\right\rangle$ for quasi-particle states with $K^{\Pi}=1 / 2^{-}, 3 / 2^{-}$, and $5 / 2^{-}$, compared to $\left\langle\Phi(q) \mid \Phi\left(q^{\prime}\right)\right\rangle$ at $\left(q+q^{\prime}\right) / 2=3000 \mathrm{fm}^{2}$

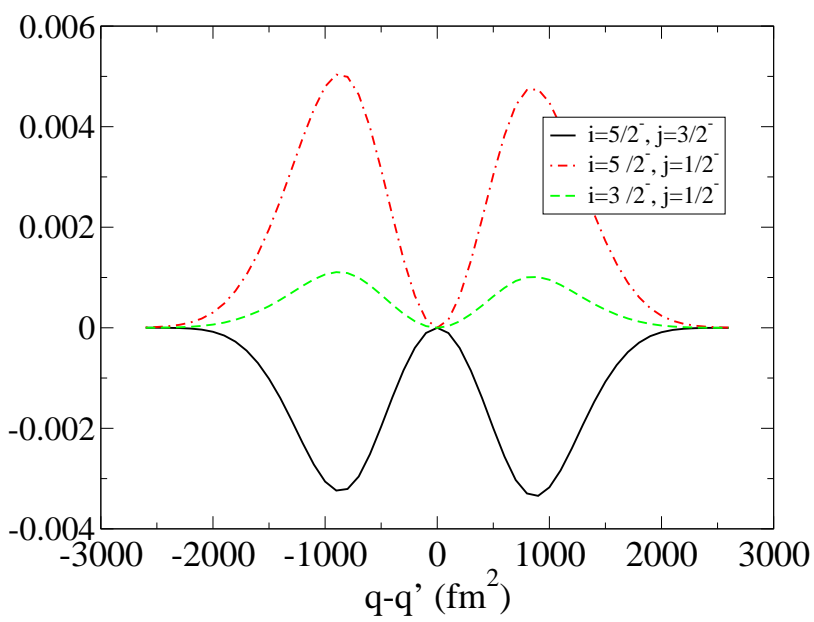

Fig. 4. (Color online) Overlaps $\left\langle\Phi_{i}(q) \mid \Phi_{j}\left(q^{\prime}\right)\right\rangle$ between different two quasi-particle excitations for $K^{\Pi}=1 / 2^{-}, 3 / 2^{-}$, and $5 / 2^{-}$at $\left(q+q^{\prime}\right) / 2=3000 \mathrm{fm}^{2}$

the development of the new formalism based on the nonadiabatic GCM.

Non diagonal overlaps $\left\langle\Phi_{i}(q) \mid \Phi_{j}\left(q^{\prime}\right)\right\rangle$ with $i \neq j$ are plotted on Fig.4. We clearly see that all non diagonal overlaps are found to be zero for $q=q^{\prime}$, as expected since 2qp states are orthogonal at a given deformation by definition. The maximum of the overlaps is predicted to be very small, namely 0.005 compared to 1 for the diagonal terms $\left\langle\Phi_{i}(q) \mid \Phi_{i}\left(q^{\prime}\right)\right\rangle$. They appear to be symmetric in a first order of approximation and have a small amplitude and an approximate sinusoidal shape.

The overlaps $\left\langle\Phi(q) \mid \Phi_{i}\left(q^{\prime}\right)\right\rangle$ are plotted on Fig. 5. The amplitudes are found to be less or equal to 0.08 , and the shape is almost antisymmetric.

In summary, all these preliminary results show that non diagonal overlaps $\left\langle\Phi_{i}(q) \mid \Phi_{j}\left(q^{\prime}\right)\right\rangle$ with $i \neq j$ are predicted to 


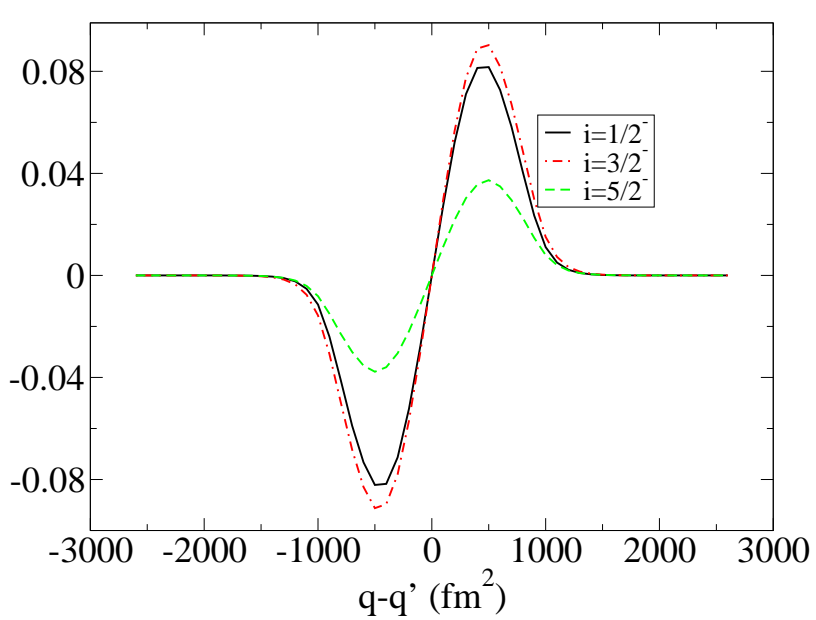

Fig. 5. (Color online) Overlaps between two quasi-particle excited states and HFB minima for $K^{\Pi}=1 / 2^{-}, 3 / 2^{-}$, and $5 / 2^{-}$at $\left(q+q^{\prime}\right) / 2=5000 \mathrm{fm}^{2}$

be much smaller than diagonal ones. A more precise study of the shape of all these overlaps is now underway.

\section{Conclusion}

A study of the role played by the intrinsic excitations along the fission paths has been undertaken. Preliminary results on norm overlaps between non-excited and excited 2 qp states have been presented in ${ }^{236} \mathrm{U}$. The next step is now to calculate the energy kernel between different basis states. On the long range, we expect to develop a fully microscopic non adiabatic formalism to improve results on fission fragment properties such as fragment charge and mass distributions.

\section{Acknowledgments}

We thank C. Simenel and J-F. Berger for helpful discussions. This work was in part performed under the Auspices of the U.S Department of Energy by the Lawrence Livermore National Laboratory under Contract DE-AC5207NA27344.

\section{References}

1. H. Goutte, J.-F. Berger, P. Casoli, and D. Gogny, Phys. Rev. C71, (2005) 024316.

2. S. Pomme et al., Nucl. Phys. A572, (1994) 237.

3. F. Vives, F.-J. Hambsch, H. Bax, and S. Oberstedt, Nucl. Phys. A662, (2000) 63.

4. S. Pomme et al., Nucl. Phys. A560, (1993) 689.

5. J.R. Nix et al., Nucl. Phys. A424, (1984) 239.

6. F. Scheuter et al., Phys. Lett. B149, (1984) 149.

7. V.M. Kolomietz, S.V. Radionov, and S. Shlomo, Phys. Rev. C64, (2001) 054302.
8. K.T.R. Davies et al., Phys. Rev. C16, (1977) 1890.

9. N. Carjan, A.J. Siek, and J.R. Nix, Nucl. Phys. A452, (1986) 381.

10. P.N. Nadtochy, A. Kelić, and K.-H. Schmidt, Phys. Rev. C75, (2007) 064614.

11. M.V. Borunov, P.N. Nadtochy, and G.D. Adeev, Nucl. Phys. A799, (2008) 56.

12. K.Dietrich, J.-J. Niez, J.-F. Berger, submitted to Nucl. Phys. A

13. N. Tajima et al, Nucl. Phys. A542, (1992) 355.

14. M. Didong, H. Müther, K. Goeke, Amand Faessler, Phys. Rev. C14, (1973) 1189.

15. H. Müther, K. Goeke, K. Allaart, Amand Faessler, Phys. Rev. C5, (1977) 1467.

16. J.-F. Berger, M. Girod, and D. Gogny, Nucl. Phys. A428, (1984) 23c.

17. P.G. Reinhard, Nucl. Phys. A261, (1976) 291.

18. P. Bonche, et al., Nucl. Phys. A510, (1990) 466.

19. J. Dechargé and D. Gogny, Phys. Rev. C21, (1980) 1568.

20. J.-F. Berger, M. Girod, and D. Gogny, Comp. Phys. Comm. 63, (1991) 365. 